\title{
Coseismic visibility of a small fragile patch involved in the rupture of a large patch - implications from fully dynamic rate-state earthquake sequence simulations producing variable manners of earthquake initiation
}

\author{
Hiroyuki Noda ${ }^{1 *}$, Masao Nakatani ${ }^{2}$ and Takane Hori ${ }^{1}$
}

\begin{abstract}
Frictional properties on a fault cannot be uniform, and one idealization would be a hierarchical asperity concept in which a large, tough patch (Patch L, radius $R^{L}$ and nucleation size $R_{c}^{L}$ ) includes small, fragile patches (Patches $S$, radius $R^{S}$ and nucleation size $R_{c}^{S}$ ), which is consistent with the scale-independent properties of earthquakes. At the onset of large earthquakes, a minor but quick signal from an immediately preceding rupture is sometimes observed during the so-called slow nucleation phase before the moment acceleration starts increasing linearly with time. Understanding what causes such seismic characteristics is important in assessing heterogeneity on a fault. In earthquake sequence simulations with hierarchical distribution of the state-evolution distance, large earthquakes spanning Patch $L$ may be initiated by cascade-up rupture growth from Patch $S$, by their own large nucleation, and by delayed cascade-up, with their occurrence ratio depending on parameters characterizing the distribution (e.g., 'scale ratio' $a=R^{L} / R^{S}$ and 'brittleness' $\beta=R^{L} / R_{c}^{L}=R^{S} / R_{c}^{S}$ ). In the present paper, we compared the coseismic moment rate and acceleration functions between different types of ruptures and between different values of $a$. The events that started from small nucleation showed quick onset in these functions compared with those from large nucleation. In a cascade-up large earthquake, a small wave from a small rupture spanning Patch $S$ preceded the main wave from the main rupture if a was larger than or comparable to $\beta$. This condition is similar to that for the appearance of small events in the simulated history that are nucleated in Patch $S$ and fail to cascade-up. If $a>>\beta$, we no longer have cascade-up large events. That is, Patch $S$ behaves as a unit of rupture for $a>>\beta$ while it merely serves as internal inhomogeneity of Patch $L$ rupture for $a<<\beta$. The transition occurs gradually with a over the intermediate range $a \sim \beta$.
\end{abstract}

Keywords: Earthquake; Rate-state friction; Hierarchical asperity

\footnotetext{
* Correspondence: hnoda@jamstec.go.jp

${ }^{1}$ Institute for Research on Earth Evolution, Japan Agency for Marine-Earth Science and Technology, 3173-25 Showa-machi, Kanazawa-ku, Yokohama 236-0001, Japan

Full list of author information is available at the end of the article
} 


\section{Background}

A nucleation phase in the seismogram is defined as a signal before the recorded ground velocity starts increasing linearly with time. Such a linear acceleration is a characteristic of self-similar rupture expansion in a three-dimensional problem. As summarized by Yoshida and Kato (2005), the nucleation phase may contain clues for understanding the fault behavior in the preseismic preparation stage. Systematic dependence on the earthquake size has been sought in the initial part of seismograms, but the results are mixed. We address them below in the context of multi-scale heterogeneity on which the present simulations are based.

A large earthquake may start from a large nucleation that is consistent with the large fracture energy observed (Venkataraman and Kanamori 2004; Kato 2012). If nucleation processes of earthquakes of different sizes can be scaled in the same manner as the coseismic processes, then a larger earthquake should have a longer nucleation phase. Some seismological studies have yielded consistent results (e.g., Iio 1992; Ellsworth and Beroza 1995; Beroza and Ellsworth 1996). Other studies (e.g., Ishihara et al. 1992; Nakatani et al. 2000) have reported that larger earthquakes show larger slopes in the nucleation phase, which indicates that the space-time scaling in the nucleation phase is different from that of the main rupture. Yet other studies have reported that the shape and amplitude of the nucleation phase do not depend on the size of the earthquakes (e.g., Mori and Kanamori 1996). Those apparently contradicting results for different datasets may represent variety in the earthquake generation processes, which might depend on geological settings, macroscopic loading processes, or some other factors.

It is occasionally reported that a minor burst in the velocity seismogram is observed during the nucleation phase (e.g., Ellsworth and Beroza 1995; Beroza and Ellsworth 1996). What kind of fault heterogeneity causes such seismic characteristics? Under what circumstances does such a minor signal appear before the main one? These are important questions aimed at understanding the potential scale-dependency of the fault friction and assessing the possibility of earthquake prediction by detecting precursory deformation.

In the hierarchical asperity concept (Ide and Aochi 2005), a larger and tougher patch (Patch L) has smaller and more fragile patches (Patches $S$ ) in it, and such a structure exists in many length scales. An assumption that 'brittleness' of the patch (i.e., size of the patches $R$ relative to their nucleation size $R_{c}$, which is proportional to the fracture energy $G_{c}$ for constant stress drop) is independent of the patch size leads to selfsimilar characteristics of earthquakes including, for example, typical rupture speeds and radiation efficiencies independent of the earthquake size (e.g., Ide and Beroza 2001; Venkataraman and Kanamori 2004). The minor burst in the velocity seismogram in the nucleation phase may correspond to the small rupture before cascadeup growth, and this has been reproduced by numerical simulations of dynamic rupture growth assuming conformance with the slip-weakening model (Ide and Aochi 2005) and the slip-strengthening-weakening model (Shibazaki and Matsu'ura 1995) with specific distributions of the parameters.

Recently, this concept was extended to the whole seismic cycle using a rate- and state-dependent friction (RSF) with hierarchical distribution of the state evolution distance $L$ (Noda et al. 2013a) (Figure 1). In such a model, two non-dimensional parameters are shown to be important in controling the behavior: one is the brittleness $\beta=R^{L} / R_{c}^{L}=R^{S} / R_{c}^{S}$ and the other is the scale ratio $\alpha=R^{L} / R^{S}$ (previously called the 'scale gap' (Noda et al. 2013a)), where superscripts $L$ and $S$ indicate Patch $\mathrm{L}$ and Patch $\mathrm{S}$, respectively. If the brittleness dominates $\left(\beta>\alpha\right.$ or $\left.R^{S}>R_{c}^{L}\right)$, then a small rupture spanning Patch $\mathrm{S}$ usually cascades up to a large earthquake and none or very few of large earthquakes are initiated by their own large nucleation (Figure 2a). If the scale ratio dominates $\left(\alpha>>\beta\right.$ or $\left.R_{c}^{L}>>R^{S}\right)$, then a rupture that has just

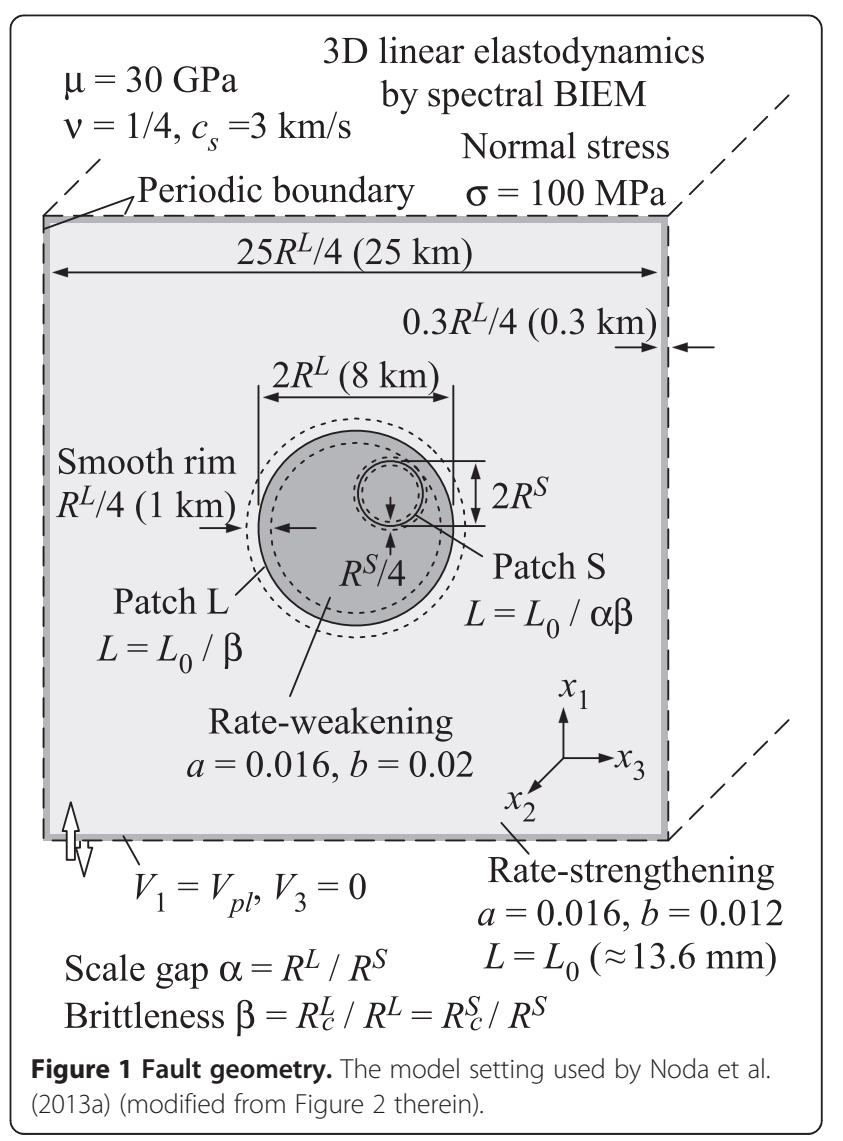




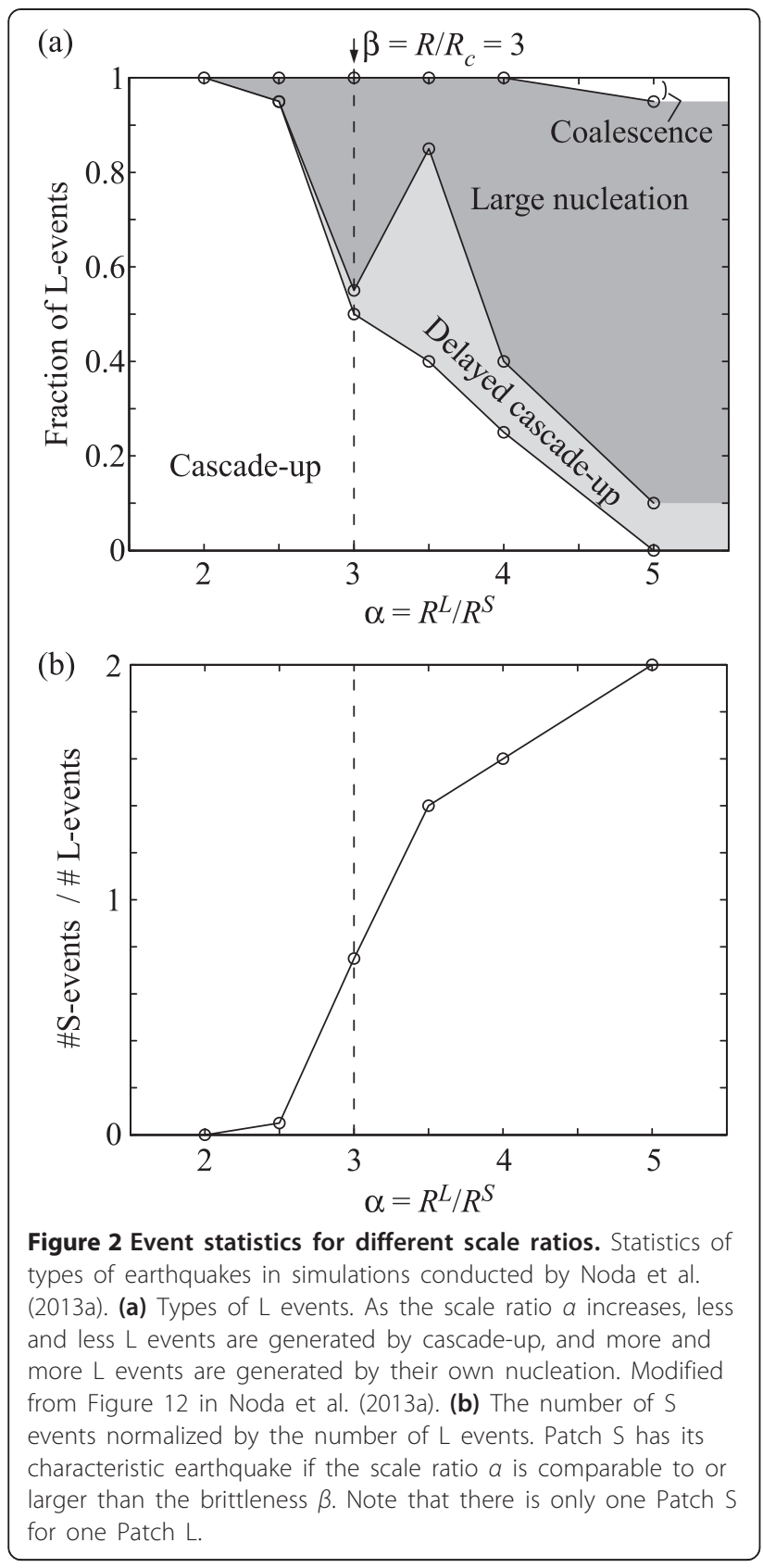

proceeded to sweep Patch $\mathrm{S}$ has too small of an energy release rate to grow further dynamically and hence ends up with a small earthquake (Figure 2b). Thus, Patch L, which cannot creep coherently because the brittleness is larger than unity, has to be ruptured by its own large nucleation (Figure 2a). If $\alpha$ and $\beta$ are comparable, then the small rupture sometimes cascades up and sometimes not, depending on the readiness of Patch $\mathrm{L}$, which is reflected in the background slip rate around Patch S. In this case, large earthquakes may be initiated either by cascade-up or by their own large nucleation in a long history.
The following interpretation, which may be obvious in a single dynamic rupture propagation with a moreor-less uniform initial condition, has also turned out to be reasonable in earthquake sequences where complicated distributions of initial stress and fracture energy for earthquakes are set spontaneously by preceding earthquake cycles. A patch with larger brittleness $\beta$ (i.e., smaller fracture energy) can rupture more easily by cascade-up from a smaller scale. Alternatively, if the fracture energy is different by a larger factor $\alpha$ between adjacent scales, then it is more difficult for a small rupture to cascade-up. The competition of $\alpha$ and $\beta$ may play an important role in determining long-term system behavior including statistics of earthquake size and manner of initiation.

The previous paper by Noda et al. (2013a) mainly reported on the abovementioned overall system characteristics and preseismic behaviors that are different between different types of earthquakes. Note that inertial effects are fully accounted for in their earthquake sequence simulations (Lapusta and Liu 2009; Noda and

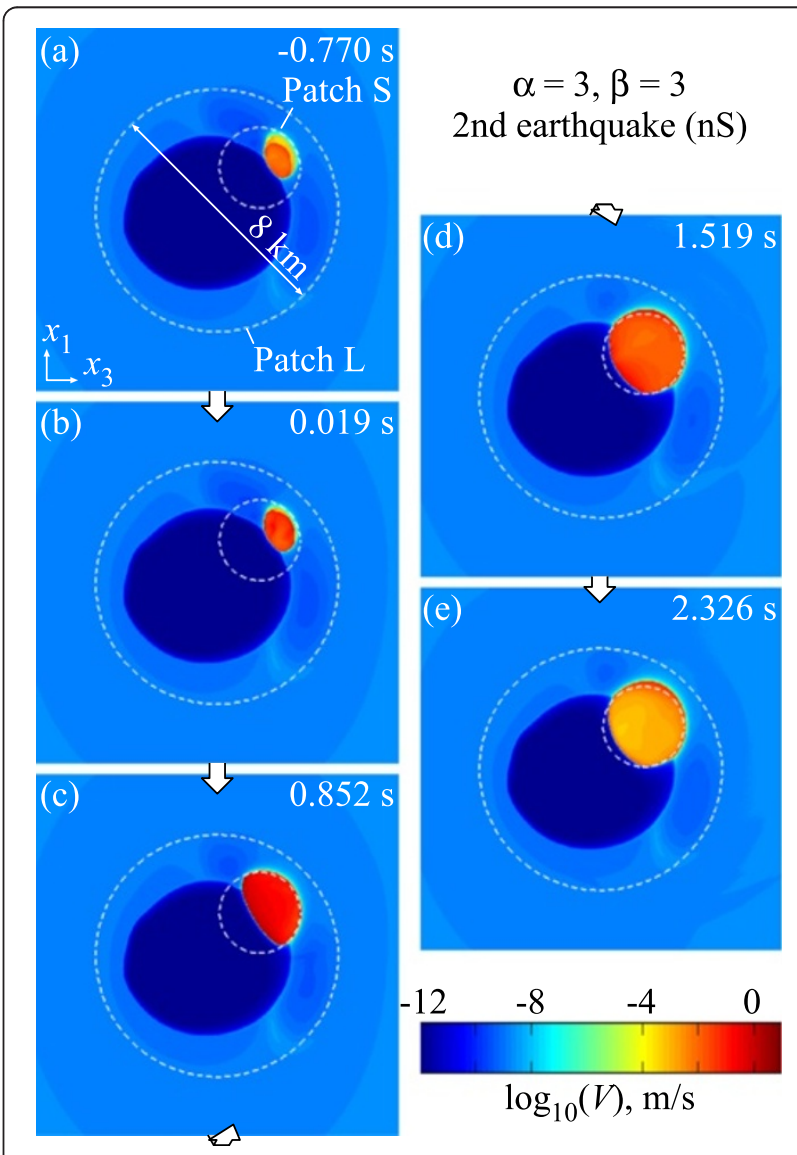

Figure $\mathbf{3} \mathbf{n s}$. (a, b, c, d, e) Snapshots of the slip rate distribution during a typical non-precursory $S$ event. $a=\beta=3$. The time after the moment acceleration exceeds a threshold $10 \mathrm{PNm} / \mathrm{s}^{2}$ is indicated in each panel. 
Lapusta 2010), and the calculated coseismic behavior is realistic in a sense that it is consistent with elastodyanamics. In the present paper, we report on the coseismic behavior of those simulations. Especially, the focus of this paper is on improving our understanding of under what circumstances do small ruptures cause minor seismic signals preceding the main one (i.e., during the nucleation phase).

\section{Methods}

The simulations discussed in the present paper have been published by Noda et al. (2013a), and extensive details about their method can be found in that study. Briefly, they conducted a series of earthquake sequence simulations using RSF with the aging law (Dieterich 1979; Ruina 1983):

$$
\begin{aligned}
& \tau=\sigma f=\sigma\left[f_{0}+\operatorname{aln}\left(\frac{V}{V_{0}}\right)+b \ln \left(\frac{V_{0} \theta}{L}\right)\right] \\
& \frac{d \theta}{d t}=1-\frac{V \theta}{L}
\end{aligned}
$$

where $\tau$ is the magnitude of the shear traction, $f$ is the friction coefficient, $\sigma$ is the normal stress, $f_{0}$ is the steady-state friction coefficient at a reference slip rate $V_{0}, V$ is the slip rate, $a$ and $b$ are non-dimensional parameters representing the amounts of the direct and evolution effect, respectively, $\theta$ is the state variable, and
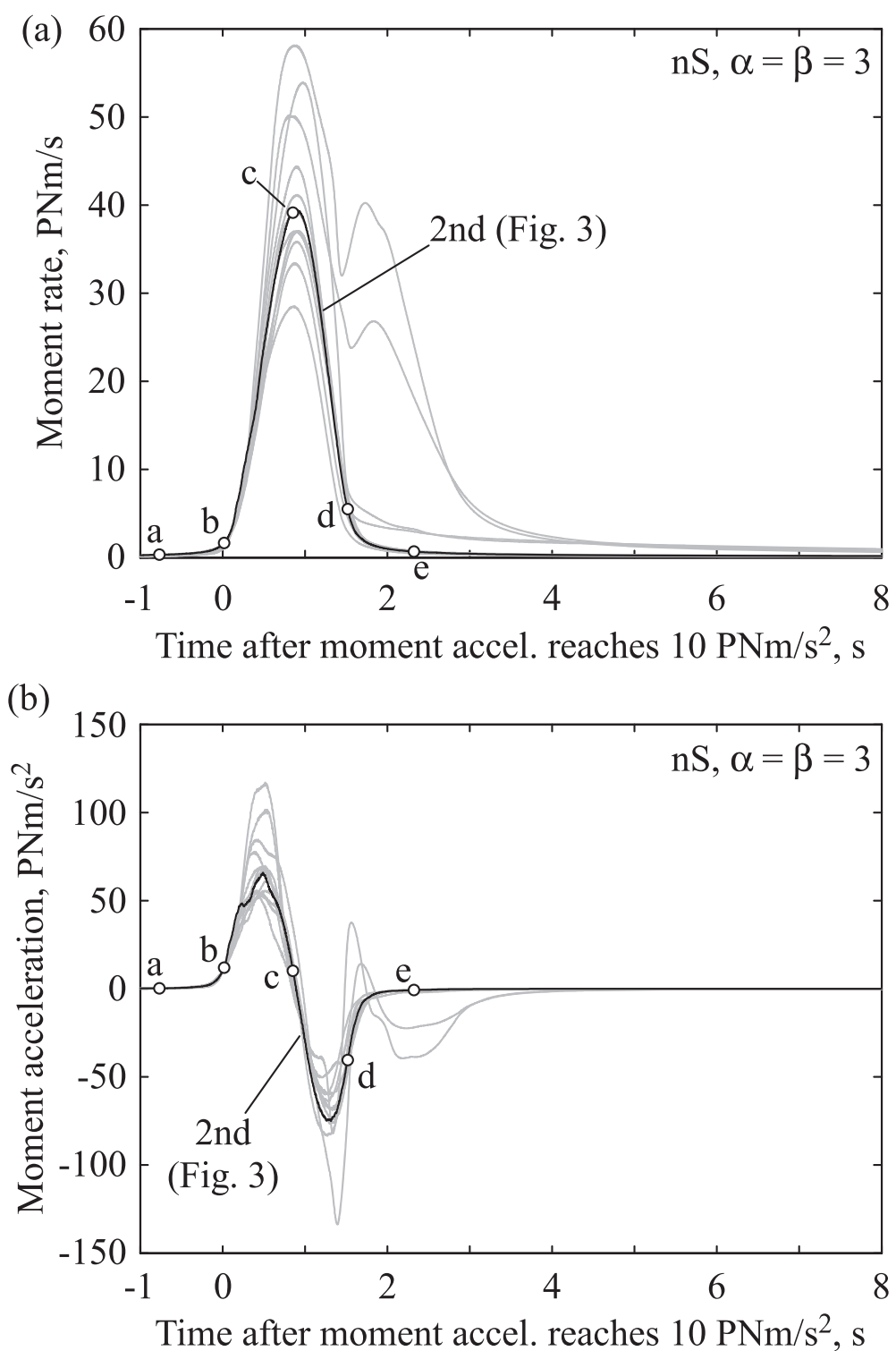

Figure 4 Moment rate and acceleration for $\mathbf{n S}$. (a) Moment rate and (b) moment acceleration functions for $\mathrm{nS}$ events where $a=3$ and $\beta=3$. The black curve is for the representative case depicted in Figure 3. The open circles labeled alphabetically indicate timings of the snapshots there. 


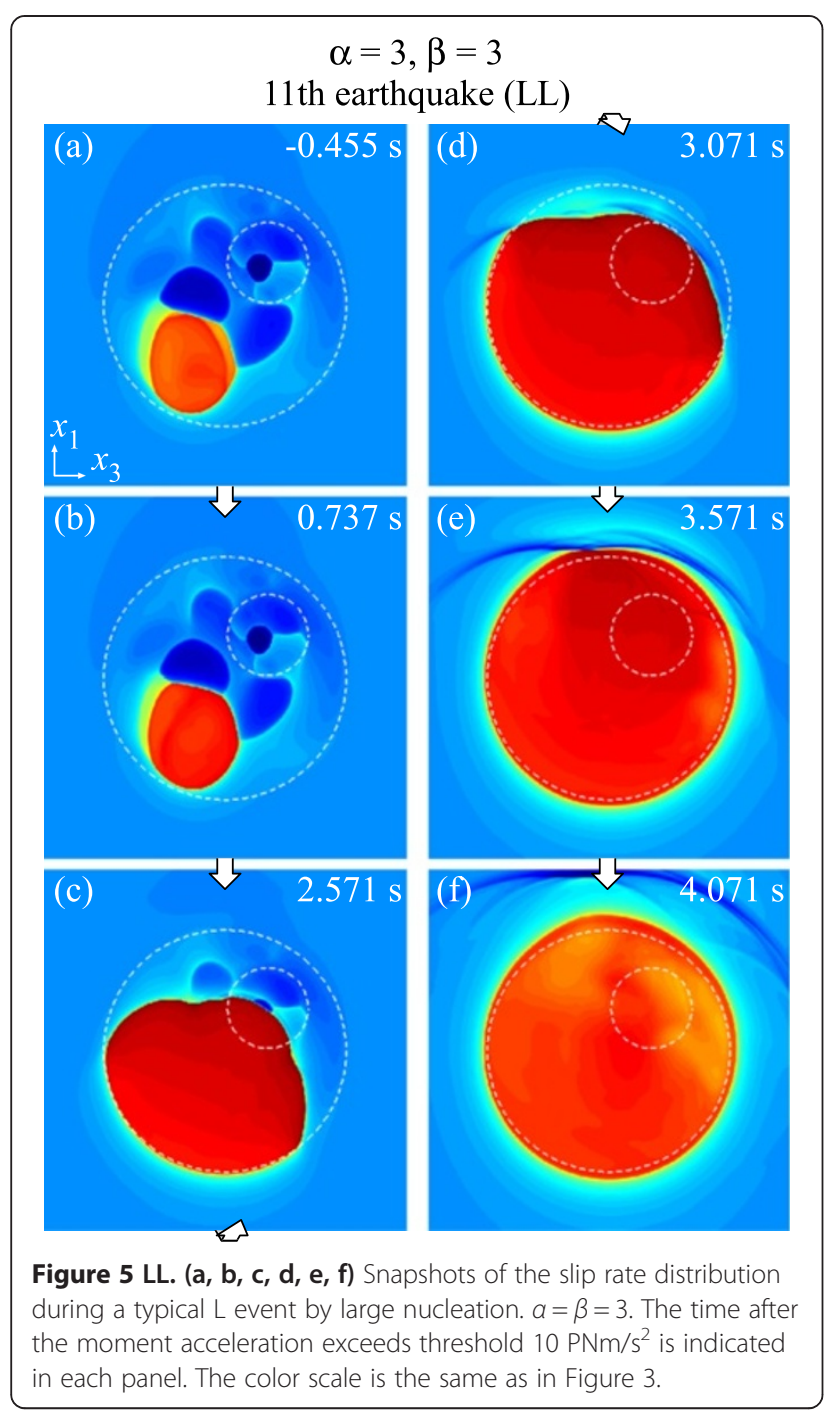

$L$ is the state-evolution distance. The parameters used are described in Figure 1. In the simulations discussed here, $\sigma=100 \mathrm{MPa}, f_{0}=0.7, V_{0}=8 \mathrm{~cm} / 3.1536 \times 10^{7} \mathrm{~s}, a=$ 0.016 , and $b$ is 0.012 outside the seismogenic patches and 0.02 in them. Note that $a / b=0.8$ in the seismogenic patches. A planar fault was embedded in a linearly elastic infinite medium with periodic boundaries (Figure 1). The elastodynamics (the shear modulus $\mu=30 \mathrm{GPa}$, the Poisson's ratio $v=1 / 4$, and the shear wave speed $c_{s}=3$ $\mathrm{km} / \mathrm{s}$ ) was treated by a spectral boundary integral method (e.g., Lapusta and Liu 2009; Noda and Lapusta 2010).

In order to investigate an elementary process in the hierarchical asperity concept, which is the interaction of two length scales different by one order in the hierarchical structure, one Patch $\mathrm{L}$ (radius $R^{L}$, nucleation radius $R_{c}^{L}$ ) having one Patch $\mathrm{S}$ (radius $R^{S}$, nucleation radius $R_{c}^{S}$ ) was considered and embedded in a rate-strengthening fault. The brittleness $\beta$ was assumed to be 3 for both
Patch L and Patch S, that is, $R^{L} / R_{c}^{L}=R^{S} / R_{c}^{S}=3$. Different scale ratios $\alpha=R^{L} / R^{S}$ ranging from 2 to 5 were investigated. For the parameters and the constitutive law used here, the nucleation radius $R_{c}$ is given approximately as (Rubin and Ampuero 2005; Chen and Lapusta 2009):

$$
R_{c}=\frac{\pi}{4} \frac{\mu b L}{\sigma(b-a)^{2}}
$$

The nucleation size is proportional to $L$. Since the brittleness is assumed to be the same for Patches $\mathrm{L}$ and $\mathrm{S}$, the values of $L$ inside those patches are proportional to the patch radii. The nucleation size which is three times smaller than the patch size in length enables initiation of earthquakes by compact acceleration, the size of which is approximately $R_{c}$ (nucleation), rather than by coalescence of creeping fronts (Chen and Lapusta 2009; Kaneko and Ampuero 2011).

The fault is loaded by prescribing a long-term slip rate $V_{p l}=V_{0}$ near the periodic boundary. The simulations were conducted until 20 large earthquakes occurred, which were approximately $\mathrm{Mw} 6$ for the Patch $\mathrm{L}$ radius $R^{L}=4 \mathrm{~km}$.

As already mentioned by Noda et al. (2013a), the scaling of the problem is relatively straightforward. With keeping the non-dimensional solution and the scales of speed and stress, an increase in the length scale (radii of the patches and $L$ ) by some factor causes an increase in the time scale by the same factor. That is, if the simulated large earthquakes are regarded as approximately $\mathrm{Mw} 8$ by saying $R^{L}=$ $40 \mathrm{~km}$ instead of $4 \mathrm{~km}$, then the time quantities (e.g., time between earthquakes and time from initiation of a dynamic rupture) must be multiplied by a factor of 10 . We selected the length scale $R^{L}=4 \mathrm{~km}$ for presentation purposes.

We would like to emphasize that modeling of successive earthquake cycles in sequence is essentially important in studying variations in the coseismic behavior. Distribution of shear stress $\tau$ and state $\theta$ at the initiation of a dynamic rupture could be regarded as the initial conditions for a dynamic rupture. If these are chosen (or tuned) arbitrarily, one may be able to 'reproduce' a variety of earthquakes, but it is difficult to judge what kind of scenarios are more likely to be the case than others.

\section{Results and discussion}

\section{Rupture processes in simulated earthquakes}

The simulations showed rich behaviors depending on the parameters $\alpha$ and $\beta$ (Figure 2). Noda et al. (2013a) classified the earthquakes that appeared in their simulations. Here, we use the same classification and discuss characteristics of the coseismic moment rate and moment acceleration functions for each class. Large (L) events are initiated by large nucleation (LL), by dynamic cascade-up rupture growth (cL), or by delayed cascade-up (dcL), 

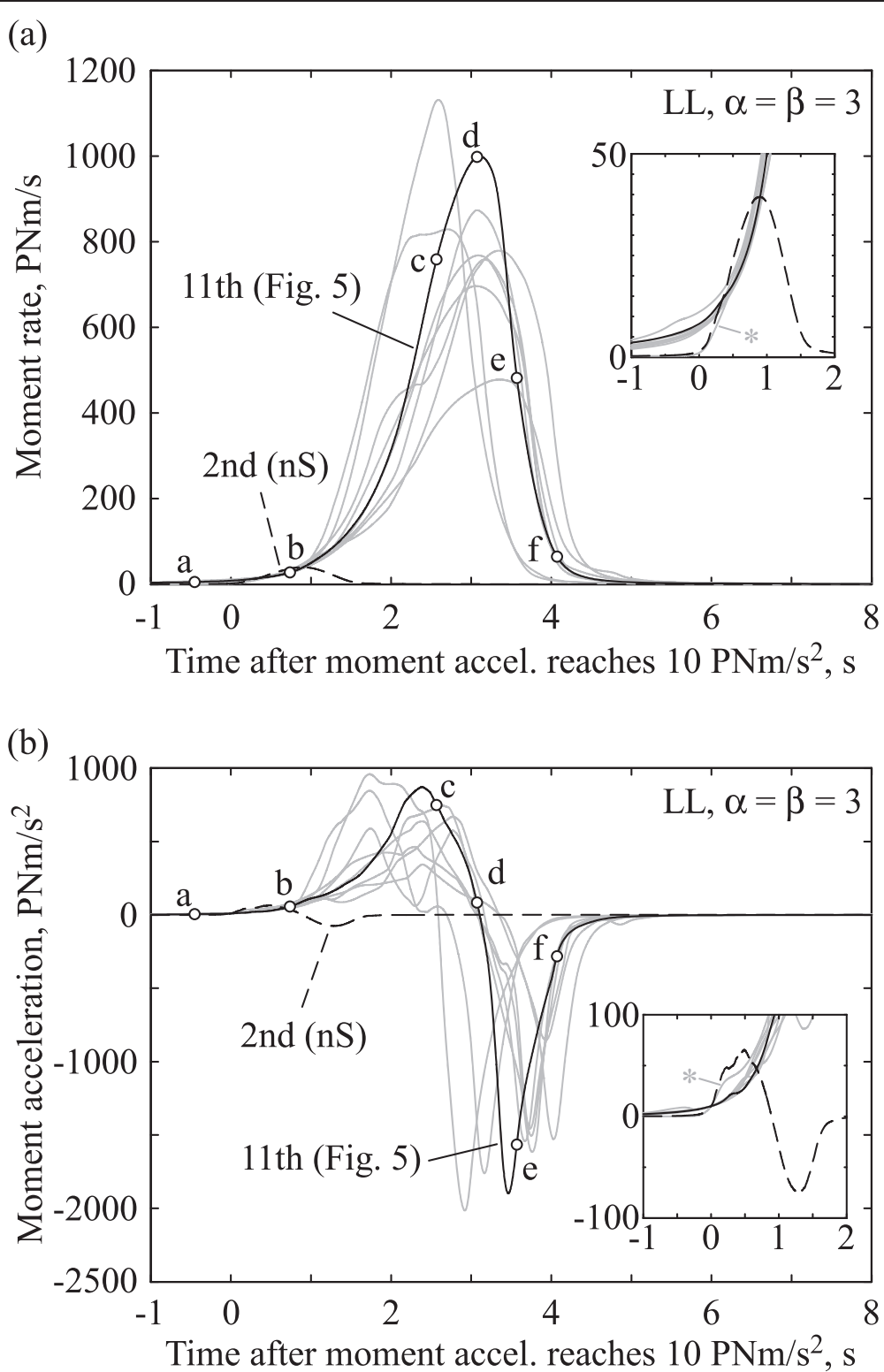

Figure 6 Moment rate and acceleration for LL. (a) Moment rate and (b) moment acceleration functions for LL events where $a=3$ and $\beta=3$. The black curve is for the representative case depicted in Figure 5. The open circles labeled alphabetically indicate timings of the snapshots there. The case with a star (9th) is duplicated in Figure 7. The 2 nd event (nS) is plotted by dashed lines for comparison.

which directly follows a preceding precursory small (pS) event. Sometimes, an LL event takes place after a pS event that has promoted the emergence of large nucleation. In addition, we obtained non-precursory small events (nS) whose afterslip decayed to the interseismic level before the occurrence of the next large earthquake. In the following subsections, we briefly review characteristics of those dynamic ruptures, which were previously reported by Noda et al. (2013a), before discussing coseismic sourcetime functions. In the next section, we shall discuss the effect of $\alpha$ and what controls the coseismic characteristics.
In Noda et al. (2013a), the earthquakes were defined by a threshold of the spatially maximum slip rate equal to $0.1 \mathrm{~m} / \mathrm{s}$. In the present paper, we used the same criterion for detecting the events so that we could use the same event catalog, but plots of moment rate and moment acceleration functions were shifted in time so that time was zero when the moment acceleration became 10 $\mathrm{PNm} / \mathrm{s}^{2} \quad\left(=10^{16} \mathrm{Nm} / \mathrm{s}^{2}\right)$ at the beginning of events in order to ease comparison. The examples shown are, unless otherwise noted, from a single simulation of a sequence of earthquakes with $\alpha=\beta=3$ in which all the 
defined earthquake types appeared before the twentieth event spanning Patch L.

\section{Non-precursory small earthquake $n S$}

After a large earthquake, which spans Patch L, it locks, and later, a creeping motion invades into it from the rim. A small nucleation within Patch $S$ takes place after the creep front reaches it (Figure 3a).

For $\alpha \geq \beta$, it is often the case that a small nucleation in Patch S (Figure 3a) initiates a rupture that spans Patch $S$ (Figure 3b,c,d) and fails to grow further dynamically (Figure 3e). This is a small earthquake (S event) caused by rupturing of Patch $S$. If the afterslip of an $S$ event settles down to the interseismic level safely, it is classified as a non-precursory $\mathrm{S}$ event $(\mathrm{nS})$. An $\mathrm{S}$ event may be shortly followed by a large event before the afterslip settles down to the interseismic level. Such an S event is classified as a precursory $\mathrm{S}(\mathrm{pS})$ event and discussed later. The preseismic moment release rate follows that for acceleration of the small nucleation dictated solely by the local frictional properties (Noda et al. 2013a), which might be the case only for the aging law (Noda et al. 2013b).

Figure 4a,b shows the moment rate and moment acceleration functions, respectively, for $\mathrm{nS}$ events calculated by spatial integration of the slip rate within Patch L. An nS event typically yields a rather simple single-peaked moment rate function and single-wiggled moment acceleration function. Note that heterogeneities in frictional properties at further smaller length scales are not considered in our simulations, and hence potential cascade-up from them, which may cause complexity in the initiation of earthquakes as discussed later, is not included in the results. Some of the $\mathrm{nS}$ events are larger than the typical ones, and these have a minor second peak in the moment rate function and unusually large amplitude of the afterslip. Those $\mathrm{nS}$ events managed to propagate a little outside Patch S, but did not cascade-up or prompt a following large earthquake.

\section{Large earthquakes initiated by large nucleation LL}

For $\alpha \geq \beta$, large earthquakes that span Patch L (L events) may be initiated by large nucleation (LL) (Figure 5). The preseismic moment release rate follows that for acceleration of the large nucleation. When the rupture front of an LLevent sweeps Patch S, it accelerates and decelerates because of the locally small fracture energy (Figure $5 \mathrm{c}, \mathrm{d}$ ), and it radiates a high-frequency wave ahead of the rupture front (Figure 5d,e,f, waves propagating to the upper-right direc tion).

LL events yield single-peaked moment rate functions (Figure 6), similarly to nS events. If we compare LL events and $\mathrm{nS}$ events when the moment acceleration reaches a threshold of $10 \mathrm{PNm} / \mathrm{s}$ (i.e., zero in the horizontal axis in
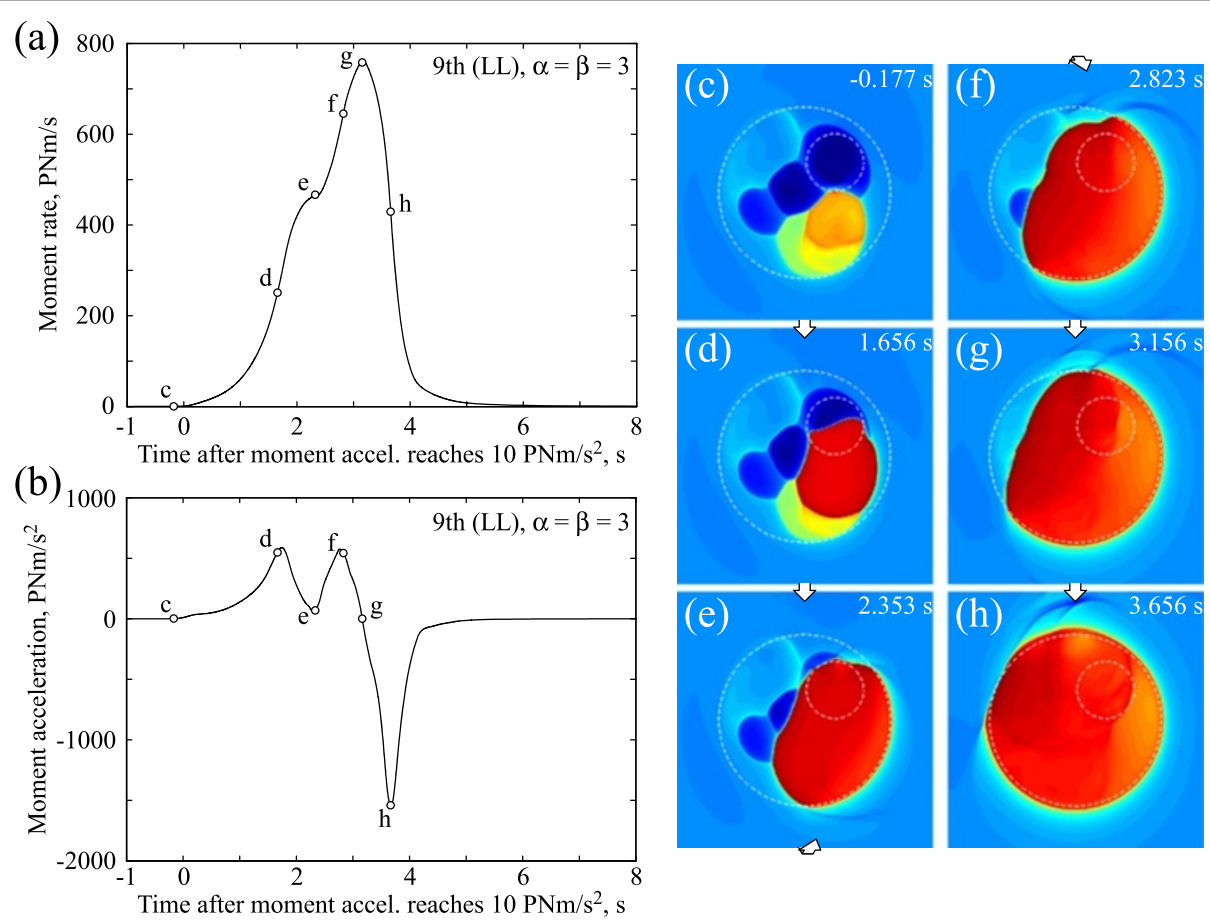

Figure 7 An LL event by interaction of large nucleation and Patch $\mathbf{S}$. The 9th earthquake with $a=\beta=3$ is shown as an example. This event is classified as an LL event because there is a large nucleation, but Patch $\mathbf{S}$ is also involved in the initiation. (a) The moment rate function is duplicated from Figure 6a. (b) The moment acceleration function is duplicated from Figure $6 \mathbf{b}$. (c, $\mathbf{d}, \mathbf{e}, \mathbf{f}, \mathbf{g}, \mathbf{h})$ Snapshots of the slip rate distribution. The time after the moment acceleration exceeds threshold $10 \mathrm{PNm} / \mathrm{s}^{2}$ is indicated in each panel. The color scale is the same as in Figure 3. 
Figure 6), LL events typically show larger moment rates and milder changes in the moment acceleration than $\mathrm{nS}$ events. If this happens in natural earthquakes, larger earthquakes will have a longer nucleation phase as Shibazaki and Matsu'ura (1998) argued. It should be noted that the absolute value of the moment acceleration is maximum for the stopping phase, which is more significant than that for $\mathrm{nS}$ events and causes non-symmetric shapes in the moment rate functions. The Patch $\mathrm{L}$ is the largest scale simulated, and hence is surrounded by a region of increased $a-b$, which acts as a stronger barrier against dynamic rupture propagation, compared with the region surrounding Patch $S$ where only $L$ is increased while $a-b$ is kept constant across the boundary (See Figure 1).

There was one exception indicated by a star in Figure 6 and duplicated in Figure $7 \mathrm{a}, \mathrm{b}$ in which the onset of the moment rate and moment acceleration functions were similar to those for $\mathrm{nS}$ events despite the fact that a large nucleation was present. This type of earthquake is initiated by interaction of ongoing large nucleation and Patch S (Figure 7c, also see Figure A5 in Noda et al. (2013a)). The dynamic rupture begins with sweeping Patch $\mathrm{S}$ and the large nucleation at the same time, which yields the first peak in the moment acceleration (Figure 7d,e). Then, the second peak in the moment acceleration appears as the rupture spans Patch $\mathrm{L}$ (Figure 7e,f,g), and this is followed by a stopping phase (Figure $7 \mathrm{~h}$ ). This example shows that an observation of short nucleation phase does not necessarily preclude operation of large nucleation. The chance of interaction between nucleation and locally fragile regions should be larger in a system with a larger number of Patches S. Investigation of such a system deserves further study.

\section{Large earthquakes initiated by cascade-up rupture growth $\mathrm{CL}$} Unless $\alpha>>\beta$, a rupture spanning Patch $S$ initiated by a small nucleation (Figure 8a,b,c) may dynamically expand outwards (Figure 8d,e) ending up with a large earthquake (Figure 8f,g,h). This is a cascade-up L (cL) event. If $\alpha \geq \beta$, a rupture spanning Patch $S$ succeeds in cascading up only when the Patch $\mathrm{L}$ is ready, and otherwise, it fails; both $\mathrm{S}$ events and $\mathrm{CL}$ events are of common occurrence in our simulations (Figure 2). Notice the difference in the overall background slip rate between Figure 3a and Figure 8a.

$\mathrm{cL}$ events are initiated from small nucleation, and thus the initial part of the moment rate and moment acceleration functions are similar to those for $\mathrm{nS}$ events (Figure 9). The amount of released moment during the initial small rupture spanning Patch $S$ is, however, larger than that in the typical $\mathrm{nS}$ event. During the main rupture spanning Patch L, it is sometimes the case that the moment acceleration increases with some complexity (for example, c to e in Figure 9b) because of heterogeneous rupture expansion
(Figure $8 \mathrm{c}, \mathrm{d}, \mathrm{e})$. The total rupture duration is longer than LL events on average, and there is larger variation.

If $\alpha<\beta$, the system falls into the one limit cycle having only one $\mathrm{cL}$ event; eventually all the earthquakes are $\mathrm{cL}$ events except just after the initiation of the simulations. The example shown in Figure 10 is for $\alpha=2.5$ and $\beta=3$. In this case, Patch $\mathrm{L}$ is ruptured by a strong perturbation (i.e., rupture of Patch $S$ ) before it ripens so that a large nucleation would occur shortly thereafter; the recurrence

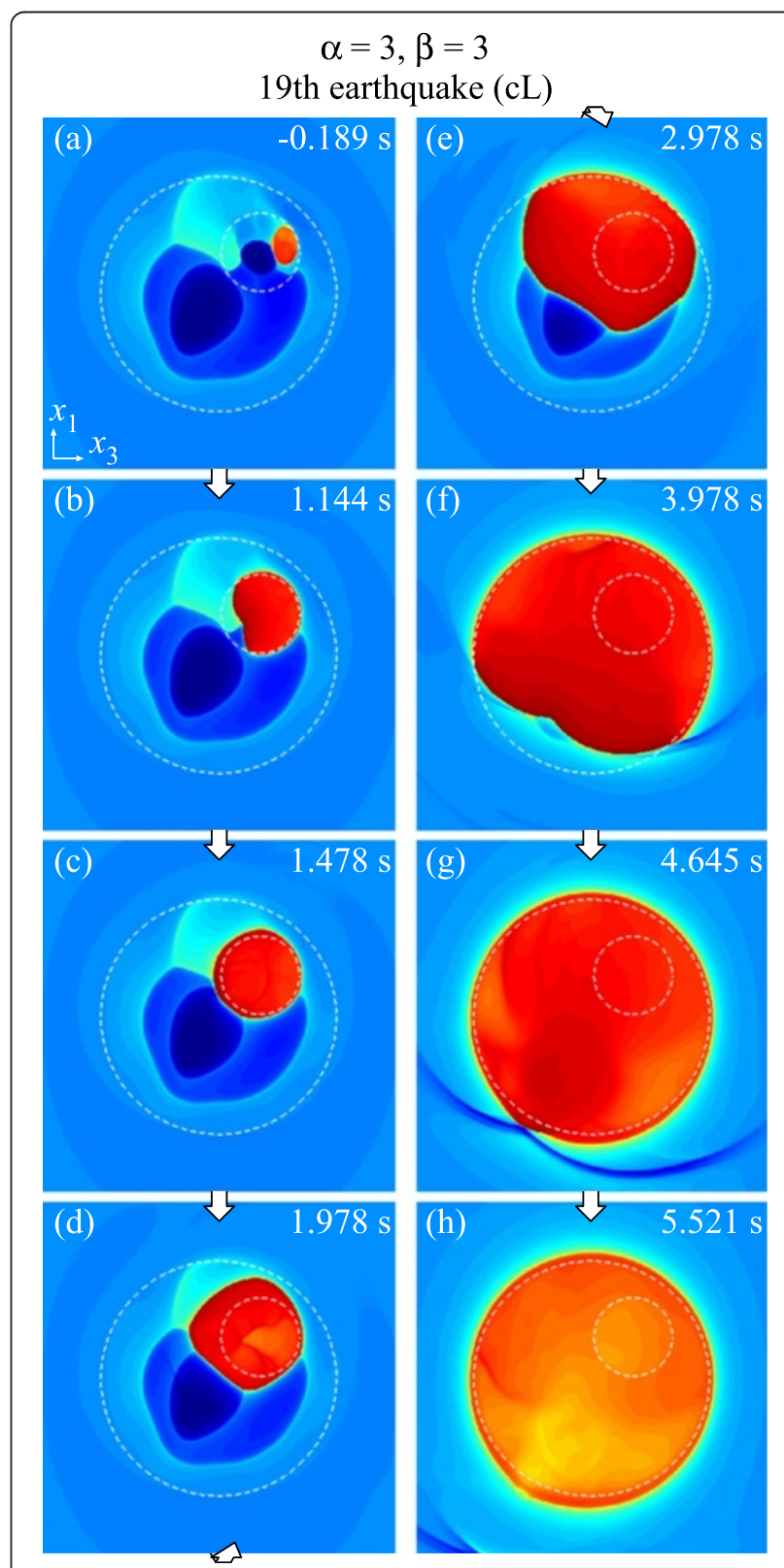

Figure $\mathbf{8} \mathrm{cL}$ for $\boldsymbol{a} \geq \boldsymbol{\beta}$. (a, b, c, d, e, f, $\mathbf{g}, \mathbf{h}$ ) Snapshots of the slip rate distribution during a typical cascade-up $L$ event where $\alpha=\beta=3$. The time after the moment acceleration exceeds threshold $10 \mathrm{PNm} / \mathrm{s}^{2}$ is indicated in each panel. The color scale is the same as in Figure 3. 

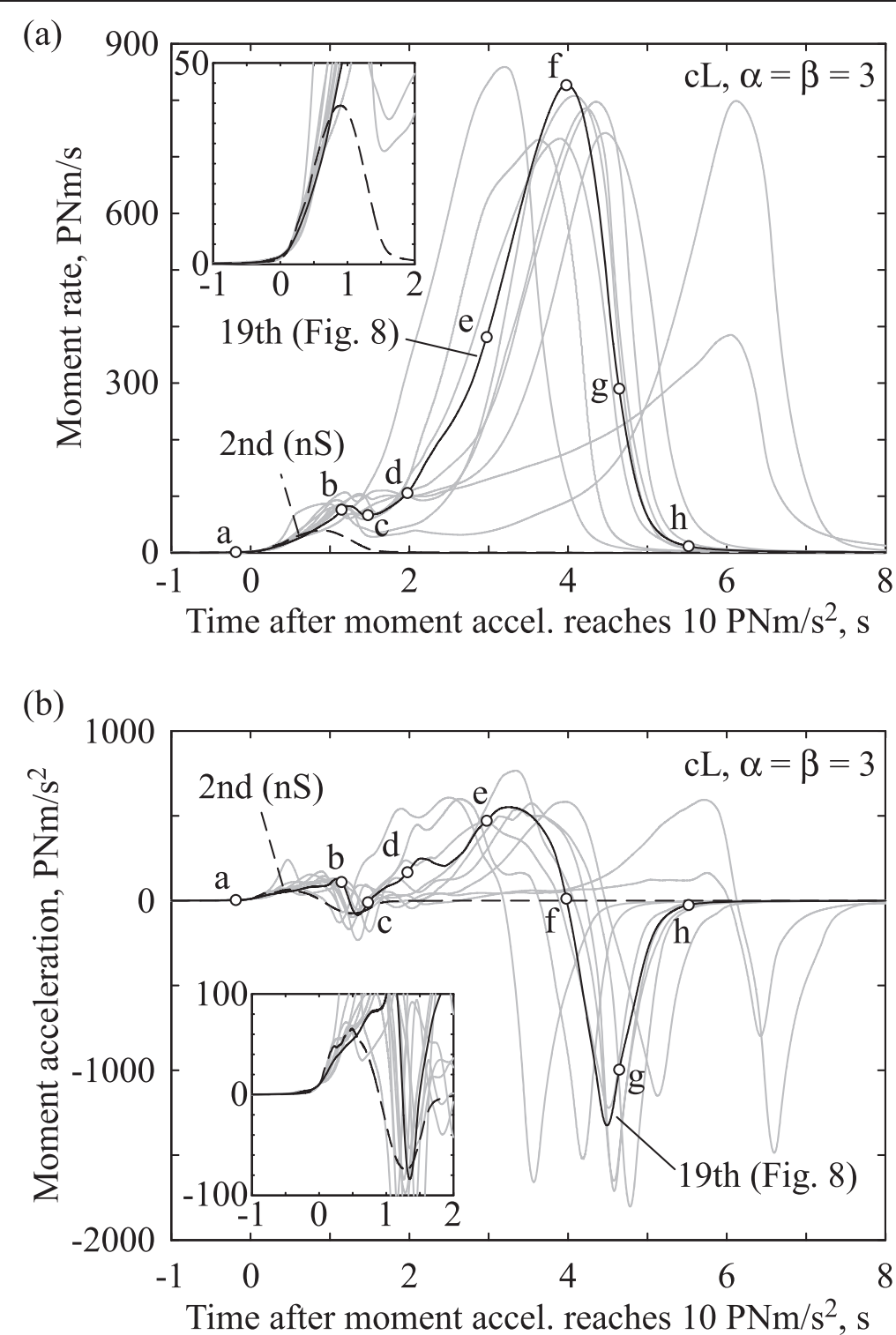

Figure 9 Moment rate and acceleration for cL. (a) Moment rate and (b) moment acceleration functions for $\mathrm{cL}$ events where $a=3$ and $\beta=3$. The black curve is for the representative case depicted in Figure 8. The open circles labeled alphabetically indicate timings of the snapshots there. The 2nd event (nS) is plotted by dashed lines for comparison.

interval of large earthquakes $(12.8 \pm 3.3$ years $)$ is significantly shorter than the case without Patch S $(17.5 \pm 3.2$ years) (Noda et al. 2013a). The rupture in such a cL event propagates unwillingly. In the example shown, the small rupture spanning Patch $\mathrm{S}$ (Figure 10a,b,c,d) is followed by bilateral (clockwise and counterclockwise) rupture propagation guided by the creeping region near the rim of Patch $\mathrm{L}$ (Figure 10e,f). The counterclockwise rupture stops, but the clockwise rupture front manages to propagate (Figure 10f, g), which supplies enough energy release rate for the counterclockwise rupture front to resume (Figure 10h). Those two rupture fronts finally meet at a point on the rim of
Patch L, but not on the opposite side from the rupture initiation because of the non-symmetric rupture process (Figure 10i,j,k). Kato (2004) suggested that by conducting quasi-dynamic simulations, such a concentration of rupture fronts may cause radiation of a seismic signal.

Figure 11 represents the moment and moment rate functions for the cL events where $\alpha=2.5$ and $\beta=3$. Note that the event with the largest moment rate is the first event that has an almost uniform preseismic shear stress and state variable. This event is not realistic in a sense that its preseismic condition is largely set by the artificially imposed initial conditions of the simulation. Later 


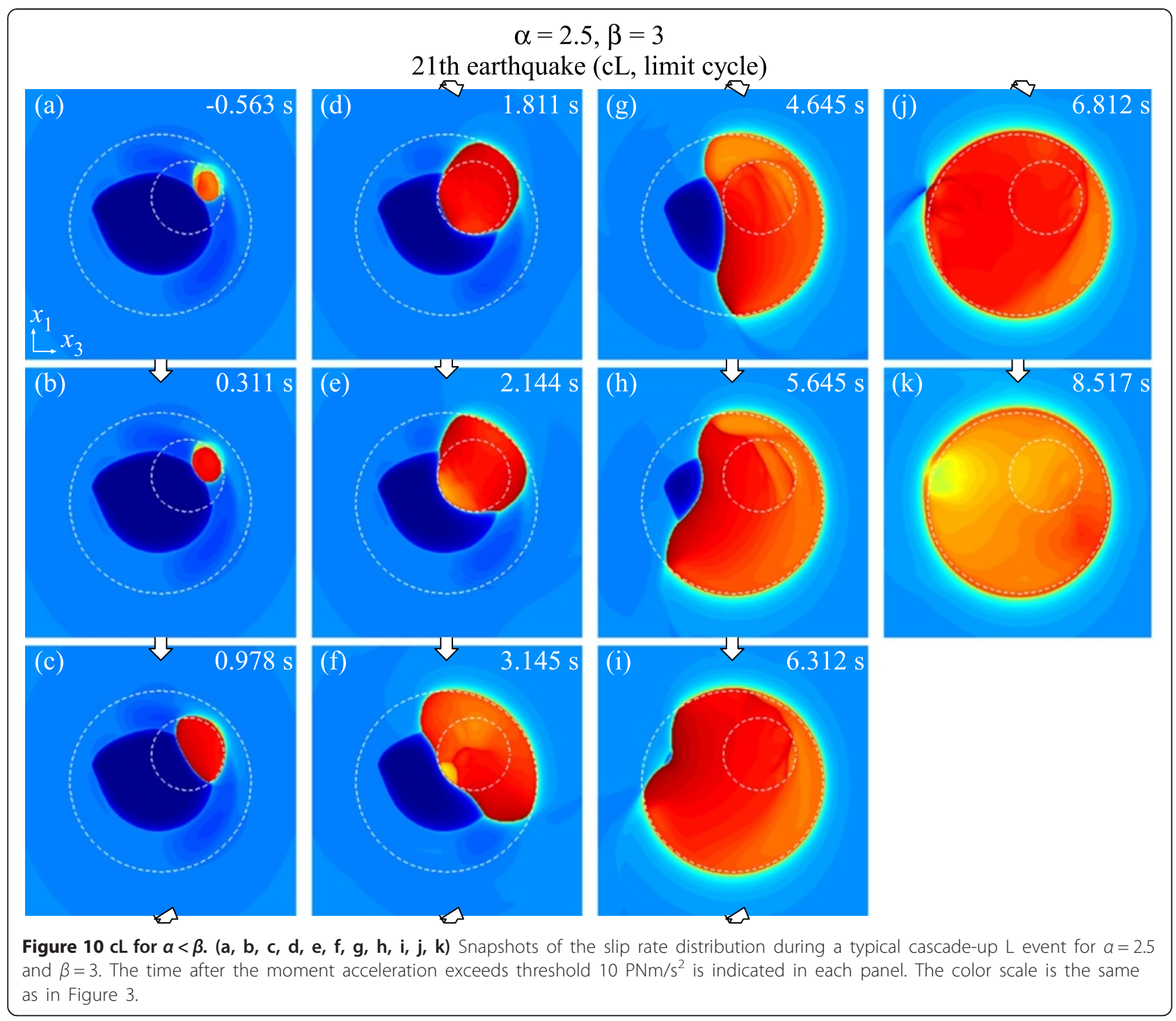

events, whose preseismic conditions are spontaneously set by interseismic processes, are dissimilar to the first one, and their source-time functions approach those of an event in a limit cycle, which we refer to as a characteristic behavior. Compared to the case with a larger scale ratio (Figure 9), rupturing of Patch S (Figure 10a,b, $\mathrm{c}, \mathrm{d})$ is not clearly reflected in the source-time functions. The rupture propagation guided by the creeping rim of Patch L (Figure 10e,f,g) is similar to long strike-slip ruptures in terms of the high aspect ratio except that the propagation direction rotates. In such a rupture, the width of the seismogenic zone gives the compliance of the system (Kanamori and Anderson 1975; Romanowicz 1992), and rupture propagation at a constant speed would cause a constant moment rate. Indeed, the moment acceleration in this stage (e to $\mathrm{g}$ in Figure 11b) is not significant. The moment acceleration takes its maximum value not during two-dimensional expansion of the rupture, but near the end of the event when the rupture fronts concentrate on the perimeter of Patch L.

The characteristics of moment rate and moment acceleration functions for cL events vary with the scale ratio as discussed later.

\section{Delayed cascade-up sequence $p S-d c L$}

An $\mathrm{S}$ event is sometimes followed shortly by an L event before the afterslip settles down to the interseismic level. Those $\mathrm{S}$ events were classified as precursory $\mathrm{S}(\mathrm{pS})$ events.

An arrested small rupture expands quasistatically (Figure 12a,b,c,d), and the following L event may be initiated by acceleration inside it including a part of the boundary of Patch S (Figure 12d,e). It expands by readvancing the arrested rupture front (Figure 12e,f), and 

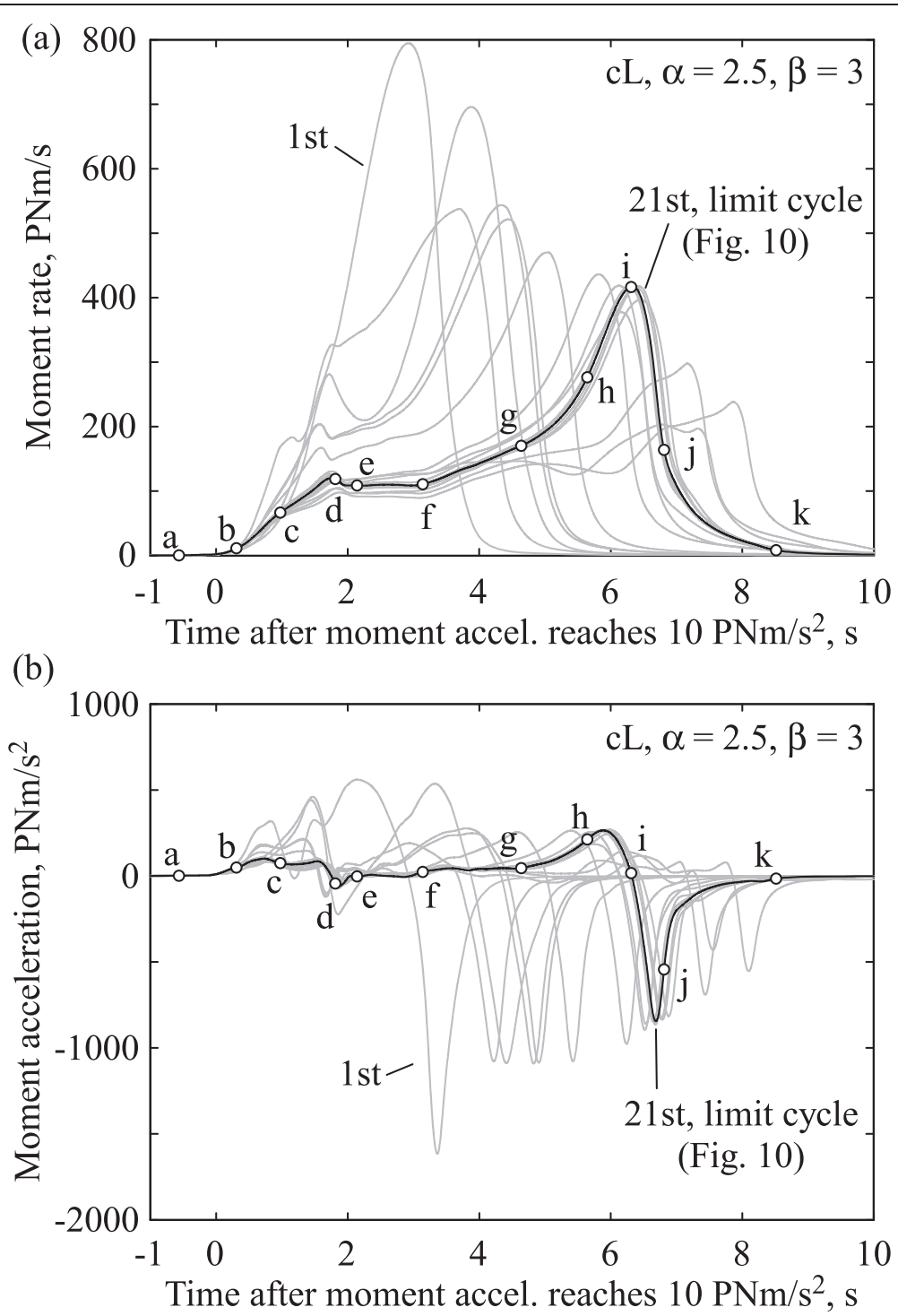

Figure 11 Moment rate and acceleration for $\mathrm{cL}$ with $\mathbf{a}=\mathbf{2 . 5}$. (a) Moment rate and (b) moment acceleration functions for $\mathrm{cL}$ events where $a=2.5$ and $\beta=3$. The black curve is for the representative case depicted in Figure 10. The open circles labeled alphabetically indicate timings of the snapshots there.

it spans Patch L (Figure 12g,h,i). Such a sequence of earthquakes can be regarded as delayed cascade-up.

Figure 13a,b represents the moment rate and moment acceleration functions during the $\mathrm{pS}-\mathrm{dcL}$ sequence, respectively. pS events tend to have larger seismic moment and afterslip than $\mathrm{nS}$ events (Noda et al. 2013a). The onset of the dcL event is characterized by milder moment acceleration similarly to an LL event. As reported by Noda et al. (2013a), the preseismic acceleration before a dcL event is similar to what is expected for a large nucleation if the time between a $\mathrm{pS}$ event and the following dcL event is long enough. As shown in Figure 13d,e, the acceleration before the dcL event takes place in a region that is somewhat smaller than the large nucleation, but definitely larger than the small nucleation. It seems then that Patch $\mathrm{S}$ just after an arrested pS event does not work as an unstable patch, and small nucleation does not occur in it.

\section{Large nucleation during an afterslip pS-LL}

An afterslip of an $\mathrm{S}$ event propagates through being guided by the creeping region inside Patch L (Figure 14a, $\mathrm{b}, \mathrm{c}, \mathrm{d}, \mathrm{e}, \mathrm{f}, \mathrm{g})$. If there is a large-enough creeping region to accommodate the large nucleation, then the afterslip may accelerate there and host a large nucleation that leads to an $\mathrm{L}$ event (Figure 14h,i,j,k,l). Similar to the $\mathrm{pS}$-dcL sequence, the background slip rate before the small nucleation tends 


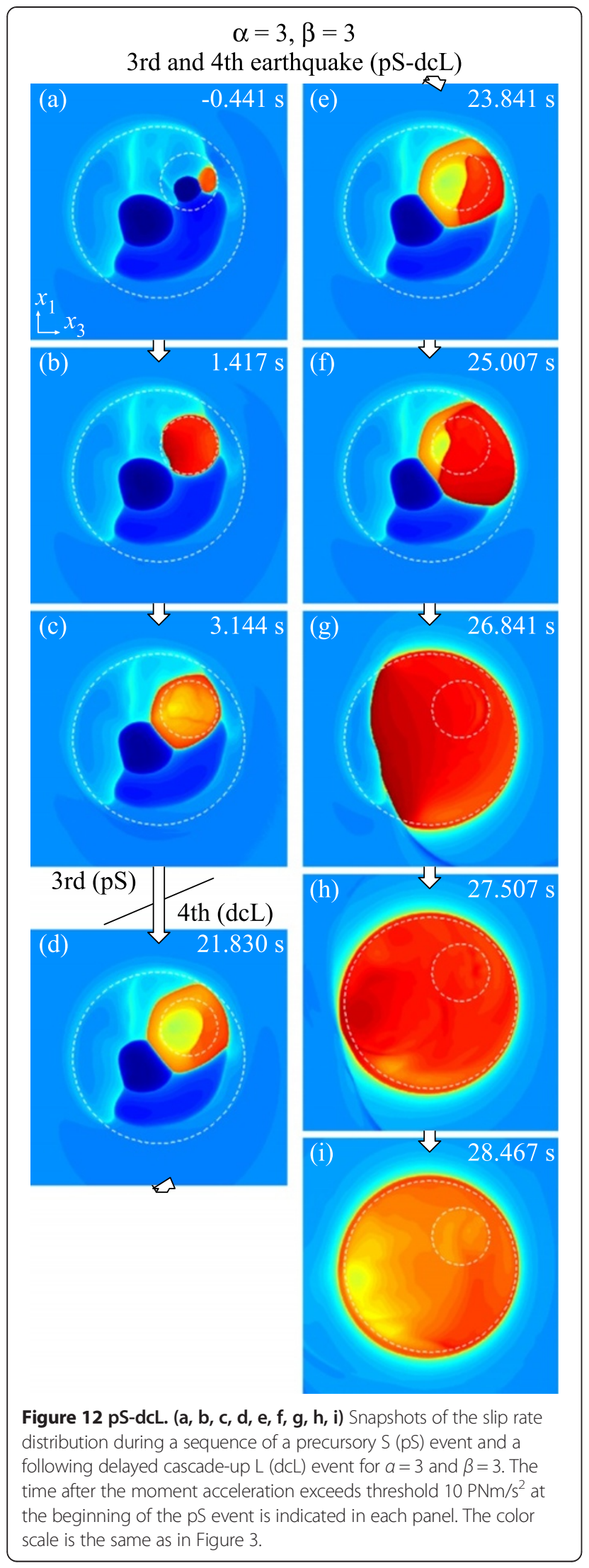

to be larger than $\mathrm{nS}$ events (Noda et al. 2013a) (compare Figures $3 \mathrm{a}$ and 14a). Additionally, pS-events before LL events tend to be larger than $\mathrm{nS}$ events (compare Figures $3 \mathrm{e}$ and 14e), similarly to $\mathrm{pS}$ events before dcL events. Figure $15 \mathrm{a}, \mathrm{b}$ shows the moment rate and acceleration functions, respectively. Compared with a typical $\mathrm{nS}$ event that ruptures the same Patch $S$, the moment acceleration is quite complex while the rupture tries to cascade-up (unsuccessfully) right after having spanned Patch $\mathrm{S}$.

There is a clear causality we can recognize between pS events and the following LL events if we can see the spatio-temporal distribution of the slip rate. Noda et al. (2013a) suggested that unusually large afterslip and its acceleration would provide hints for detecting this type of earthquake sequence, although a clear threshold in the bulk afterslip (moment rate) amplitude does not exist.

\section{Brittleness as a threshold in the scale ratio}

Cascade-up rupture growth is usually considered as a series of discrete step-by-step processes in which we observe 'a small wave from a small rupture followed by a large wave from a large rupture' (e.g., Ellsworth and Beroza 1995; Ide and Aochi 2005). Figure 16 shows the moment rate and acceleration functions of cL events for different $\alpha$ from 2 to 4 together with a typical LL event (the 11th event with $\alpha=3$ shown in Figure 6) in gray dashed lines for reference. Our parameter study about the scale ratio $\alpha$ has demonstrated that the chance for a large earthquake to show such stereotypical behavior is high under a condition where the scale ratio $\alpha$ is comparable to the brittleness $\beta$. Too small $\alpha$ causes almost all the $\mathrm{L}$ events to be initiated by cascade-up, but the signal from Patch $\mathrm{S}$ is difficult for us to recognize as a discrete small preceding wave. For example, in the case with $\alpha=2$ in Figure 16, the height of the positive peak in the moment acceleration is larger for the rupture of Patch $S$ than for the rupture of the rest. A continuous distribution of $L$ that increases proportionally with the distance from the center of Patch $\mathrm{L}$ can be regarded as a limiting case of $\alpha \rightarrow 1$ from above. Such is not "hierarchical' any more, but it still is consistent with the self-similar rupture growth with a constant rupture speed. As $\alpha$ increases, the coseismic behaviors of the cL events become apparently more consistent with the stereotypical behavior and a peak in the moment rate for the rupture of Patch $S$ becomes more distinguishable. Too large of an $\alpha$ will cause most of the large earthquakes to be initiated by their own nucleation; we no longer obtain a cL event with $\alpha=5$ at least within our simulated history worth of 20 cycles of L events (Noda et al. 2013a) (Figure 2).

With increasing $\alpha$, Patch S starts having its characteristic earthquakes from around $\alpha \sim \beta$ (Figure $2 \mathrm{~b}$ ). This condition is similar to that for the small wave preceding $\mathrm{CL}$ events to become distinguishable from the large main one. The scale 

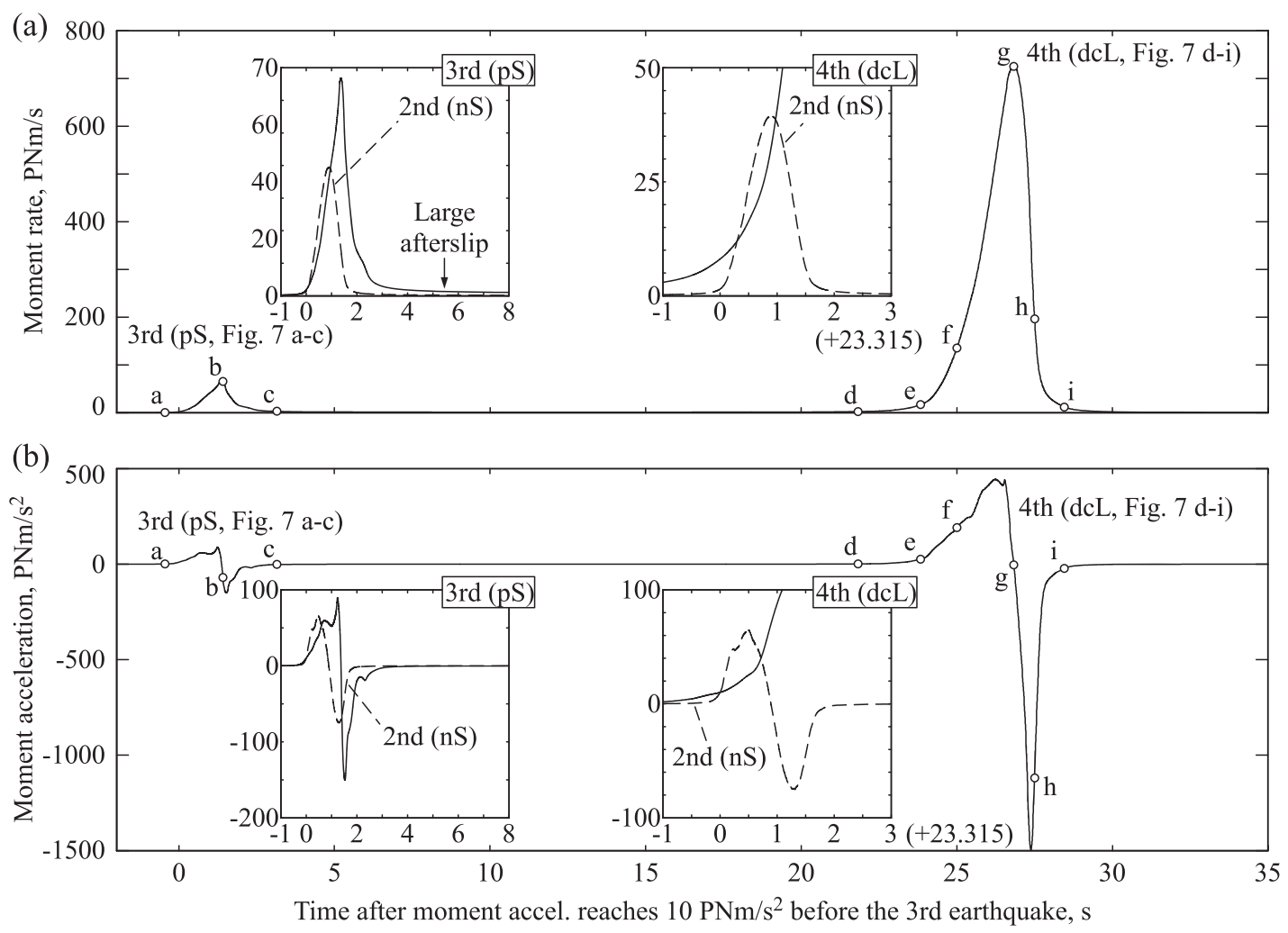

Figure 13 Moment rate and acceleration for pS-dcL. (a) Moment rate and (b) moment acceleration functions for a sequence of pS-dcL. At the beginning of the dcL-event, the moment acceleration exceeds threshold $10 \mathrm{PNm} / \mathrm{s}^{2}$ at $21.315 \mathrm{~s}$ in the horizontal axis. The 2nd event (nS) is plotted by dashed lines for comparison.

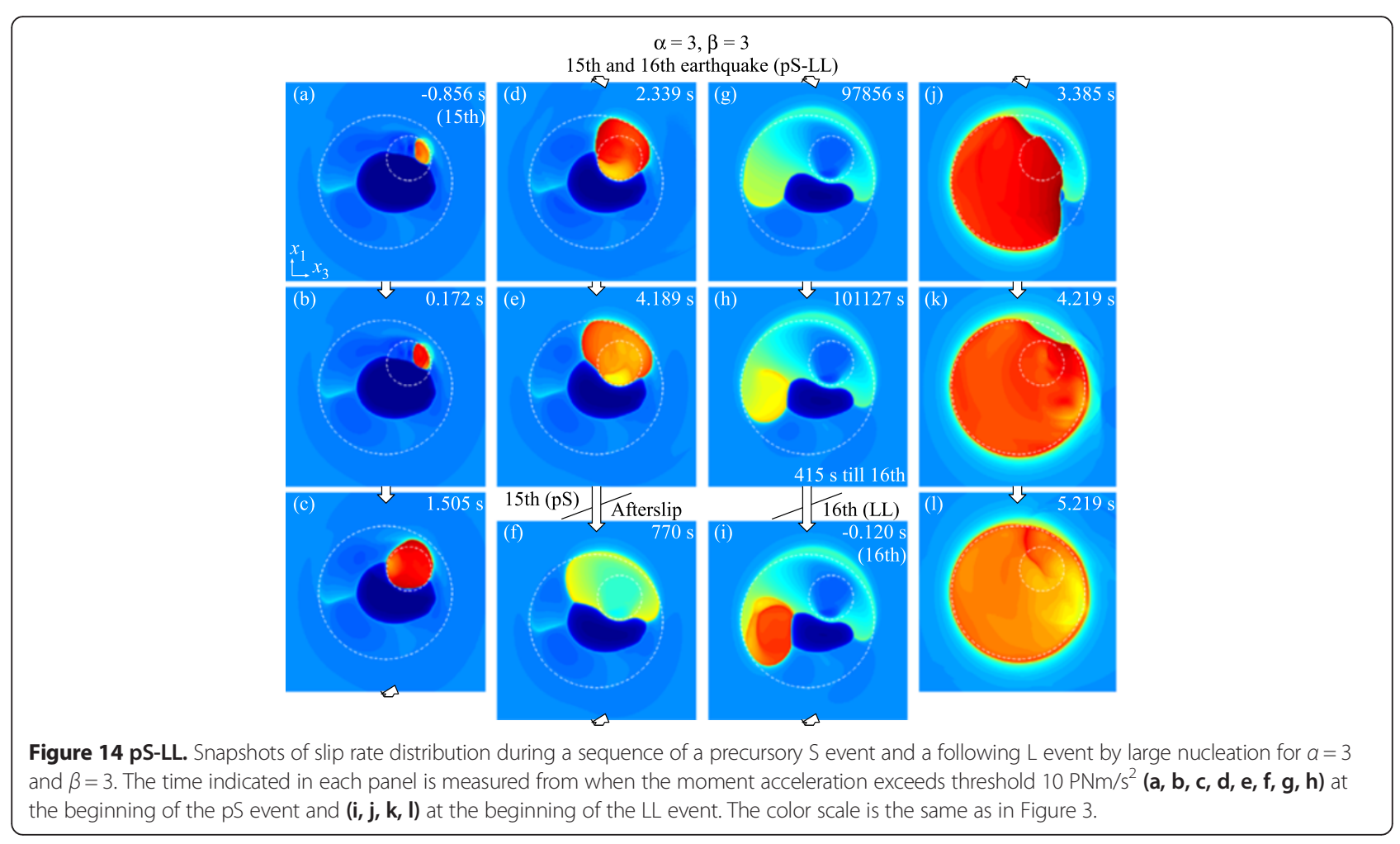



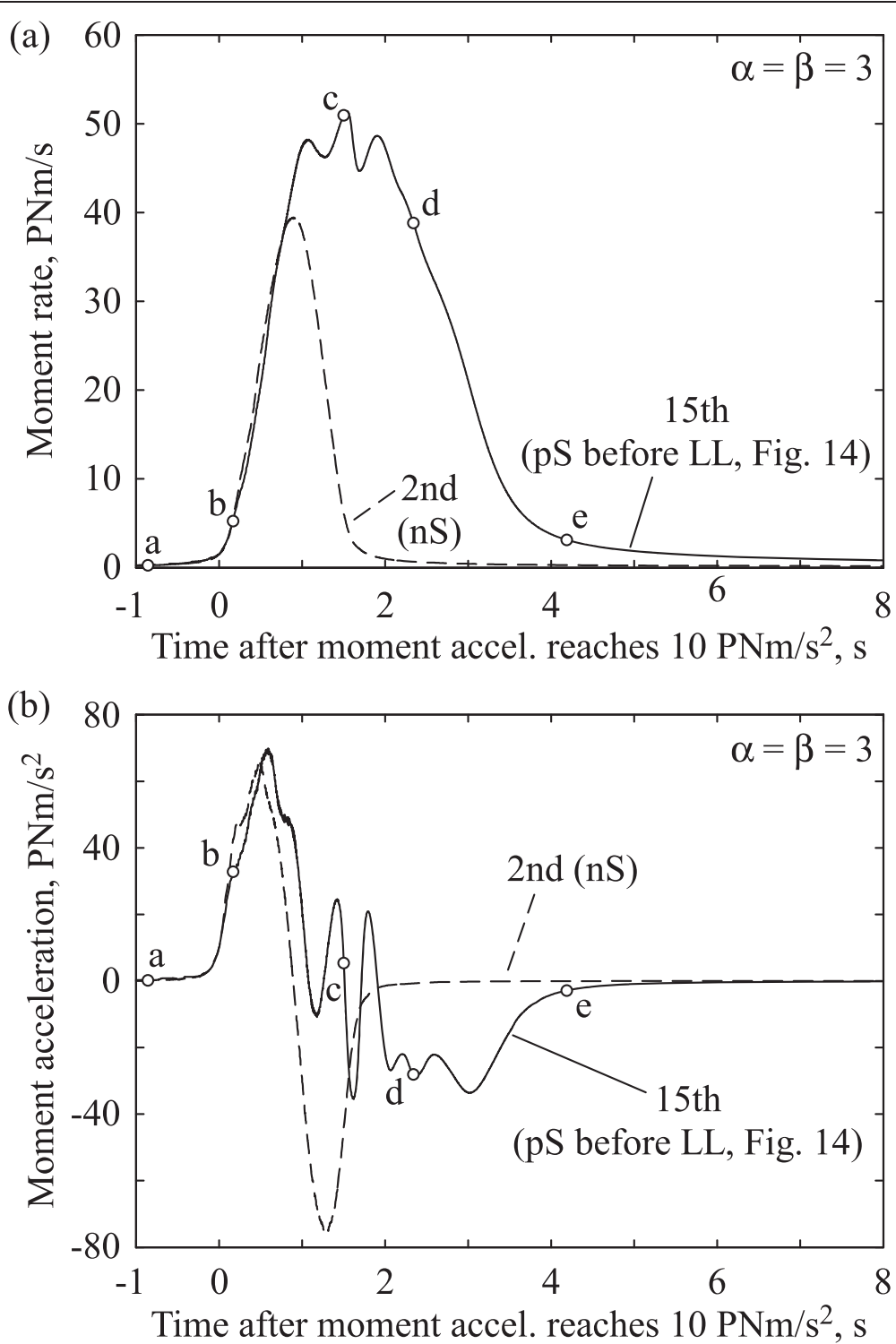

Figure 15 Moment rate and acceleration for pS before LL. (a) Moment rate and (b) moment acceleration functions for the pS event before the $\mathrm{LL}$ event shown in Figure 14.

ratio $\alpha$ represents a factor by which the fracture energy increases when a rupture cascades-up. It affects whether a rupture spanning Patch S successfully cascadesup or not, and if successful, how much the rupture speed and thus moment release decelerate through the equation of motion of the rupture front (Freund 1990). The nucleation size in the simulated cases can be estimated from the condition for the energy release rate of the expansion of the nucleus being equal to the fracture energy. Let us consider an ideal case where $\alpha$ is slightly smaller than $\beta$ and the heterogeneous preseismic conditions are neglected. In such a case, a rupture nucleated in Patch $\mathrm{S}$ spans it, decelerates to near-zero rupture speed at the rim of Patch $S$, and again spontaneously grows outwards. If Patch $\mathrm{S}$ has so small a scale ratio that it does not have its characteristic earthquake and a rupture spanning, it always cascades-up, then it is not working as a unit of rupture and would be better considered as an internal structure of Patch $\mathrm{L}$ rather than a discrete patch. The brittleness $\beta$ may give a threshold in the scale ratio $\alpha$ in order for a fragile inclusion to work as a discrete patch in a larger patch.

The location of the Patch $S$ is another important factor. Even if $\alpha>\beta$, the Patches $\mathrm{S}$ which are far enough from the rim of Patch $\mathrm{L}$ cannot interact with the invading creep front, and hence, a small nucleation is not produced in them. We tried a preliminary simulation (data not shown) 


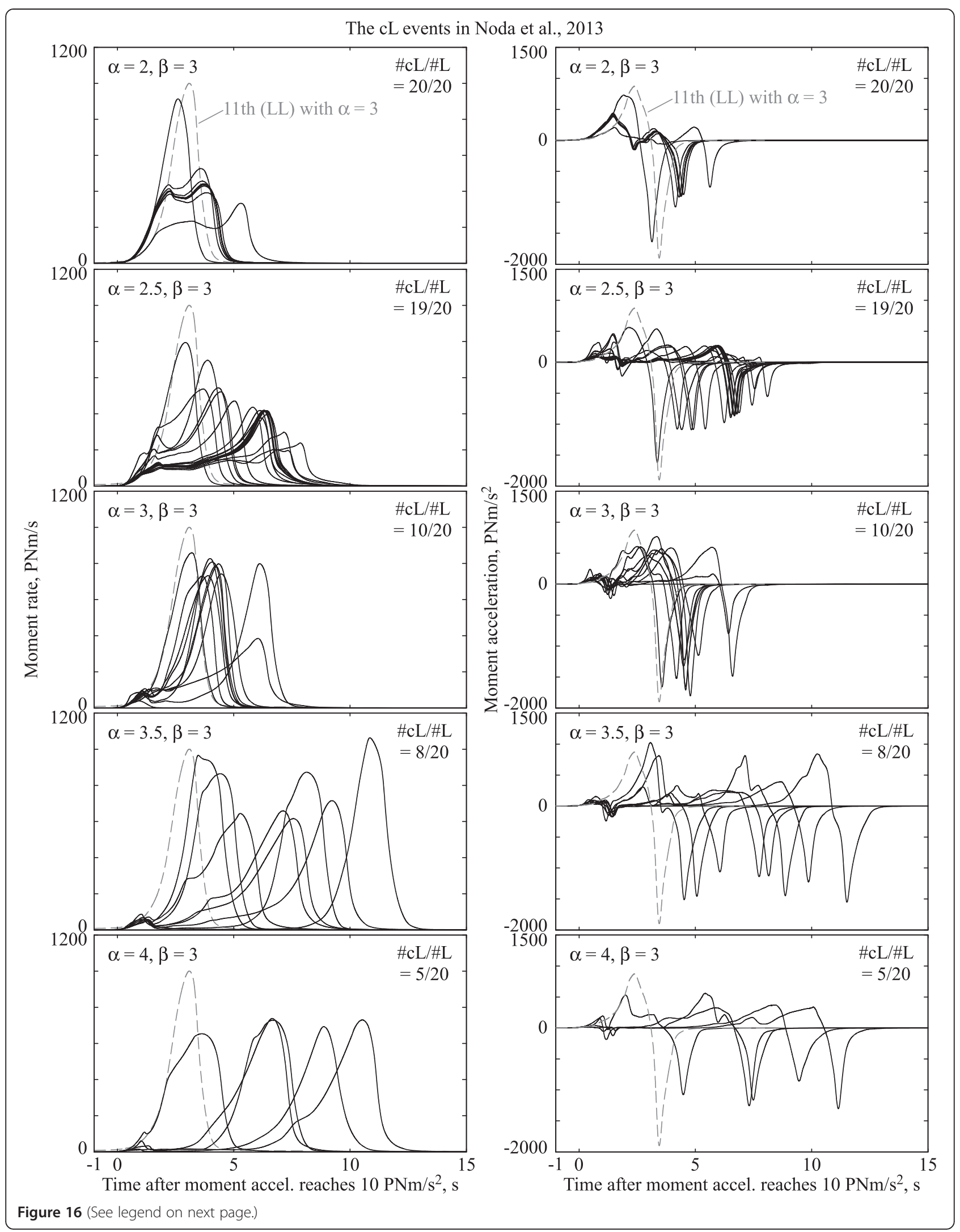


with Patch S at the center of Patch L. During the rupture of Patch L, local acceleration of the rupture front occurred at Patch S, but like in the LL event plotted in Figure 6, this behavior was not clearly recognized in the source-time function, though seismic analyses with spatial resolution, such as back projection or waveform inversion, may be able to see it.

It should be noted that the range of the scale ratio we have simulated may not be wide enough to extrapolate the abovementioned observation of the simulated results to natural faults. What if there are many tiny fragile patches spaced densely enough so that a large nucleation would necessarily include or interact with them? Such a simulation is numerically very expensive and has not been done yet, but we speculate that an ongoing large nucleation may host many tiny earthquakes that would look like clustered foreshocks (Ohnaka 1993; Shibazaki and Matsu'ura 1995; McLaskey and Kilgore 2013). Those fragile patches inside a large nucleation may also act as sources of the small preceding waves within the nucleation phase of the main rupture. Although the resulting seismogram may have a similarity to the $\mathrm{CL}$ events with large $\alpha$ demonstrated in the present study, a large nucleation (or preslip) would be present there and produce the small ruptures as a side-effect. Such behavior is not regarded as the cascade-up (Dodge et al. 1996).

\section{Conclusions}

Noda et al. (2013a) conducted earthquake sequence simulations with a rate- and state-dependent friction law and modeled a large, tough patch (Patch L) with a small, fragile patch (Patch S) in it in order to realize the hierarchical asperity concept and to investigate the elementary process involving the interaction between the scales. They reported on the characteristics of the overall system behavior as well as the preseismic processes for different scale ratios (ratio between the radii of the patches). In the present paper, the coseismic behavior from the same simulations was reported. Note that the inertial effects were fully accounted for in the simulations (Lapusta and Liu 2009; Noda and Lapusta 2010).

Earthquakes initiated by small nucleation in Patch $\mathrm{S}$ showed a quicker increase in the moment rate and acceleration at the onset of seismograms than those by a large nucleation in Patch L. If the nucleation size of Patch $\mathrm{L}$ is comparable to the size of Patch $\mathrm{S}$, then large earthquakes spanning the same Patch L may be generated by the small nucleation and grow via cascade-up (cL), by delayed cascade-up (dcL), or by the large nucleation (LL) in a long simulated history. dcL events, which shortly follow precursory small events $(\mathrm{pS})$, showed milder onset than cL events.

The cascade-up rupture growth is sometimes considered as a series of discrete step-by-step process in which we can observe 'a small wave from a small rupture followed by a large wave from a large rupture' (e.g., Ellsworth and Beroza 1995; Ide and Aochi 2005). Such idealization may be valid only when the scale ratio $\alpha$ is comparable to the brittleness $\beta$ (ratio between the patch size and the nucleation size). If $\alpha<\beta$, eventually all the earthquakes are cL events, but the rupture of Patch $S$ is difficult to recognize in moment rate or moment acceleration functions as a discrete step preceding the main part of the rupture. If $\alpha>>\beta$, then all the large earthquakes are initiated by their own large nucleation.

The condition $\alpha \sim \beta$ is a threshold for the appearance of small events ( $\mathrm{S}$ events), which are nucleated in Patch $\mathrm{S}$ and arrested after spanning it. In the hierarchical asperity concept (Ide and Aochi 2005) in which the brittleness $\beta$ is independent of the patch size and thus consistent with the self-similar characteristics of the earthquakes (e.g., Ide and Beroza 2001; Venkataraman and Kanamori 2004), the scale ratio $\alpha$ represents the factor of the fracture energy increment that must be overcome for successful cascadeup rupture growth. Even if a rupture cascades-up, the increase in the fracture energy causes a decrease in the rupture speed and thus deceleration of the moment release. Patch $\mathrm{S}$ tends to operate as a discrete patch for large $\alpha$, and its critical value seems to be given by the brittleness $\beta$.

Interaction of the large nucleation and tiny patches, which may rupture as a side-effect of the acceleration of the large nucleation, would cause clustered foreshocks as observed for some earthquakes (Ohnaka 1993; Shibazaki and Matsu'ura 1995). This is another possibility for generating the small preceding wave, but the large rupture in this class may not be able to be called a cascade-up rupture. Such a system has not been investigated partly because of the burdensome requirements for computational resources, but it deserves future study.

\section{Abbreviations}

$\mathrm{CL}$ : large event initiated by dynamic cascade-up rupture growth; dcL: large event initiated by delayed cascade-up; LL: large event initiated by large nucleation; nS: non-precursory small event; pS: precursory small event.

\section{Competing interests}

The authors declare that they have no competing interests. 


\section{Authors' contributions}

HN designed and conducted numerical simulations using the Earth Simulator as a collaborator of $\mathrm{TH}$, and $\mathrm{HN}$ analyzed the computational results. MN and $\mathrm{TH}$ suggested implementation of the hierarchical asperity concept into the rate-state earthquake sequence simulations. All the authors contributed in the discussions. HN took the lead in composing the manuscript, and $\mathrm{MN}$ and $\mathrm{TH}$ contributed to it as well. All authors read and approved the final manuscript.

\section{Acknowledgements}

We appreciate the recommendation by T. Matsuzawa, one of the session conveners of the $2013 \mathrm{JpGU}$ annual meeting, for the submission of the present study to PEPS. The Earth Simulator was used for the simulations. This study was partially supported by the Observation and Research Program for the Prediction of Earthquakes and Volcanic Eruptions of the Ministry of Education, Culture, Sports, Science and Technology (MEXT) of Japan.

\section{Author details}

${ }^{1}$ Institute for Research on Earth Evolution, Japan Agency for Marine-Earth Science and Technology, 3173-25 Showa-machi, Kanazawa-ku, Yokohama 236-0001, Japan. Earthquake Research Institute, The University of Tokyo, 1-1-1 Yayoi, Bunkyo-ku, Tokyo 113-0032, Japan.

Received: 8 January 2014 Accepted: 15 April 2014

Published: 12 May 2014

\section{References}

Beroza GC, Ellsworth WL (1996) Properties of the seismic nucleation phase. Tectonophysics 261:209-227

Chen T, Lapusta N (2009) Rate and state friction laws can explain scaling of small repeating earthquakes. J Geophys Res 114:B01311. doi:10.1029/2008JB005749

Dieterich JH (1979) Modeling of rock friction: 1: experimental results and constitutive equations. J Geophys Res 84(B5):2161-2168

Dodge DA, Beroze GC, Ellsworth WL (1996) Detailed observations of California foreshock sequences: implications for the earthquake initiation process. J Geophys Res 101(B10):22-371-22-392. dio:10.1029/96JB02269

Ellsworth WL, Beroza GC (1995) Seismic evidence for an earthquake nucleation phase. Science 268:851-855

Freund LB (1990) Dynamic fracture mechanics. Cambridge University Press, Cambridge

Ide S, Aochi H (2005) Earthquakes as multiscale dynamic ruptures with heterogeneous fracture surface energy. J Geophys Res 110:11303. doi:10.1029/2004JB003591

Ide S, Beroza GC (2001) Does apparent stress vary with earthquake size? Geophys Res Lett 28(17):3349-3352

lio Y (1992) Slow initial phase of the P-wave velocity pulse generated by microearthquakes. Geophys Res Lett 19(5):477-480. doi:10.1029/92GL00179

Ishihara Y, Fukao Y, Yamada I, Aoki H (1992) Rising slope of moment rate functions: the 1989 earthquakes off east coast of Honshu. Geophys Res Lett 19:873-876

Kanamori H, Anderson DL (1975) Theoretical basis of some empirical relations in seismology. Bull Seismol Soc Am 65(5):1073-1095

Kaneko Y, Ampuero J-P (2011) A mechanism for preseismic steady rupture fronts observed in laboratory experiments. Geophys Res Lett 38:21307. doi:10.1029/2011GL049953

Kato N (2004) Interaction of slip on asperities: numerical simulation of seismic cycles on a two-dimensional planar fault with nonuniform frictional property. J Geophys Res 109:12306. doi:10.1029/2004JB003001

Kato N (2012) Fracture energies at the rupture nucleation points of large interplate earthquakes. Earth Plant Sci Lett 353-354:190-197. doi:10.1016/j. epsl.2012.08.015

Lapusta N, Liu Y (2009) 3D boundary-integral modeling of spontaneous earthquake sequences and aseismic slip. J Geophys Res 114:09303. doi:10.1029/2008JB005934

McLaskey GC, Kilgore BD (2013) Foreshocks during the nucleation of stick-slip instability. J Geophys Res 118(6):2982-2997. doi:10.1002/jgrb.50232

Mori J, Kanamori H (1996) Initial rupture of earthquakes in the 1995 Ridgecrest, California sequence. Geophys Res Lett 23(18):2437-2440

Nakatani M, Kaneshima S, Fukao Y (2000) Size-dependent microearthquake initiation inferred from high-gain and low-noise observations at Nikko district, Japan. J Geophys Res 105:28,095-28,109

Noda H, Lapusta N (2010) Three-dimensional earthquake sequence simulations with evolving temperature and pore pressure due to shear heating: effect of heterogeneous hydraulic diffusivity. J Geophys Res 115:12314. doi:10.1029/2010JB007780

Noda H, Nakatani M, Hori T (2013a) Large nucleation before large earthquakes is sometimes skipped due to cascade-up - implications from a rate and state simulation of faults with hierarchical asperities. J Geophys Res 118. doi:10.1002/jgrb.5021

Noda H, Nakatani M, Hori T (2013b) A slower fault may produce a smaller preseismic moment rate: non- $1 / \mathrm{t}_{\mathrm{f}}$ acceleration of moment rate during nucleation and dependency on the background slip rate. Geophys Res Lett 40:4850-4854. doi:10.1002/grl.50962

Ohnaka M (1993) Critical size of the nucleation zone of earthquake rupture inferred from immediate foreshock activity. J Phys Earth 41:45-56

Romanowicz B (1992) Strike-slip earthquakes on quasi-vertical transcurrent faults: inferences for general scaling relations. Geophys Res Lett 19(5):481-484

Rubin AM, Ampuero J-P (2005) Earthquake nucleation on (aging) rate and state faults. J Geophys Res 112:11312. doi:10.1029/2005JB003686

Ruina A (1983) Slip instability and state variable friction laws. J Geophys Res $88: 10,359-10,370$

Shibazaki B, Matsu'ura M (1995) Foreshocks and pre-events associated with the nucleation of large earthquakes. Geophys Res Lett 22(10):1305-1308

Shibazaki B, Matsu'ura M (1998) Transition process from nucleation to high-speed rupture propagation: scaling from stick-slip experiments to natural earthquakes. Geophys J Int 132:14-30

Venkataraman A, Kanamori H (2004) Observational constraints on the fracture energy of subduction zone earthquakes. J Geophys Res 109:05302. doi:10.1029/2003JB002549

Yoshida S, Kato N (2005) Dependence of preslip on eventual earthquake size. Zisin 58(2):231-246. In Japanese with English abstract

doi:10.1186/2197-4284-1-8

Cite this article as: Noda et al.: Coseismic visibility of a small fragile patch involved in the rupture of a large patch - implications from fully dynamic rate-state earthquake sequence simulations producing variable manners of earthquake initiation. Progress in Earth and Planetary Science 2014 1:8.

\section{Submit your manuscript to a SpringerOpen ${ }^{\odot}$ journal and benefit from:}

- Convenient online submission

- Rigorous peer review

- Immediate publication on acceptance

- Open access: articles freely available online

- High visibility within the field

- Retaining the copyright to your article

Submit your next manuscript at $>$ springeropen.com 